\title{
Particle swarm optimization (PSO)-based node and link lifetime prediction algorithm for route recovery in MANET
}

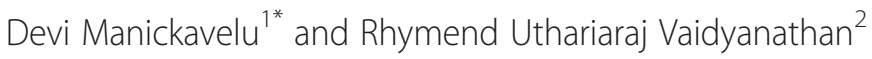

\begin{abstract}
In the conventional mobile ad hoc network (MANET) systems' route rediscovery methods, there exists route failure in all route discovery methods resulting in data loss and communication overheads. Hence, the routing has to be done in accordance with mobility character of the network. In this manuscript, a particle swarm optimization (PSO)-based lifetime prediction algorithm for route recovery in MANET has been proposed. This technique predicts the lifetime of link and node in the available bandwidth based on the parameters like relative mobility of nodes and energy drain rate, etc. Using predictions, the parameters are fuzzified and fuzzy rules have been formed to decide on the node status. This information is made to exchange among all the nodes. Thus, the status of every node is verified before data transmission. Even for a weak node, the performance of a route recovery mechanism is made in such a way that corresponding routes are diverted to the strong nodes. With the aid of the simulated results, the minimization of data loss and communication overhead using PSO prediction has been discussed in detail.
\end{abstract}

\section{Introduction}

\subsection{MANET}

Mobile ad hoc network (MANET) is a multihop wireless network with mobile nodes that can move independently. MANET has no infrastructure in the sense that it does not require any access points or base stations for transmission [1]. Nodes can communicate with each other directly or through intermediates [2]. As the nodes move arbitrarily in the network, the network topology can change frequently. One node will communicate with the other node directly within sufficient radio propagation and indirectly through multihop routing with all other nodes. To help such kind of communication, many routing algorithms already have been developed. In MANET, the nodes are randomly present and they are supposed to develop and maintain the entire network automatically; hence, the routing algorithms are crucial. Due to active topology and limited resources, developing a dynamic routing protocol that can efficiently find a routing path with low control overhead is very important in MANETs [3-5]. Most of the devices and systems in

\footnotetext{
* Correspondence: devimanickaveluphd@gmail.com

${ }^{1}$ Research Scholar, Department of Information \& Communication Engineering, Anna University, Chennai-25, India

Full list of author information is available at the end of the article
}

MANET are designed in a performance-oriented manner, not considering the energy efficiency [6].

\subsection{Significance of node and link lifetime prediction for route recovery process}

In general, the network depends on the node assistance for providing the packet routing. Routing is the basic operation in ad-hoc networks. The routing algorithm should be robust, adaptive, and in a self-organized way [5,7]. Nodes cannot forward the data packets to the receiver node when the prediction error is less than a pre-configured threshold value. Prediction is used to make the decision for transmission [8]. A. Vasilakos et al. [9] have presented an application of evolutionary-fuzzy prediction in inter-domain routing of broadband network connections with quality-of-service requirements in the case of an integrated ATM and SDH networking architecture.

The node mobility increases the complexity of routing because the greater the mobility of the nodes, the more chances of link breakage. This breakage will in turn lead to increased routing control overhead and will reduce the efficiency of the network due to the increased frequency of the route discovery process. Hence, the 
action of link breakages in MANET becomes a vital factor. Further, this kind link breakage will also lead to frequent path failures and may cause route reconstructions. As a result, the overhead of the routing protocol will be increased and the lesser packet delivery ratio and longer end-to-end delay will be terminated $[4,5]$. Re-routing in a mobile ad-hoc network is costly and would result in flooding the network due to the lack of infrastructure [10]. In addition to that, the re-route discovery is also leading to the large control message overhead and high latency. Therefore, the re-route discovery reduces efficiency of the networks [11]. Routing in MANET is restricted by the network breakage due to their node mobility or energy depletion of the mobile nodes $[12,13]$.

The existing MANET routing protocols do not operate well in environments prone to frequent and long-lived disruptions. These routing protocols assume usually connected network and require an end-to-end path to exist in order for a source to send data to a destination [14]. Nodes lie near the station are often included in the routing path. Hence, the energy of the node drains quickly [15].

\subsection{Salient feature of particle swarm optimization}

Besides computational intelligence (CI) [16], artificial intelligence techniques are nowadays involved in various applications. Several studies make use of genetic algorithm (GA)-based techniques to solve network problems [17]. Particle swarm optimization (PSO) is a stochastic optimization technique developed by the inspiration of the social behavior of bird flocking or fish schooling. In PSO, each single solution is a 'bird' in the search space (particle). The strength value is combined with each particle, which is calculated by the fitness function to be optimized, and it has the velocity, which expresses the flying of the particle. The particles will fly in the search space and will adjust with the velocities dynamically according to their historical behaviors. This process will guide the particles to fly toward the better search area in the search space. In MANET, the work of sending the packets from source to destination is difficult because of the mobility of the elements and there is no central control. To solve these problems, the swarm intelligence concept can be applied. The PSO algorithm was initially introduced by Kennedy and Eberhart (1995) in terms of social and cognitive behavior. This technique resolves the problems in various fields such as engineering and computer science [18-20].

\section{Related works}

Xin Ming Zhang et al. [5] have proposed an estimated distance (EstD)-based routing protocol (EDRP) to guide a route discovery. This protocol can restrict the propagation range of route request and reduce the routing overhead.
The change regularity of the received signal strength is exploited to estimate the geometrical distance between a pair of nodes, which is called the estimated geometrical distance (EGD). An estimated topological distance (ETD) is a topology-based EstD that can mitigate the effect of inaccurate EGD. The EstD is a combination of EGD and ETD. Every node evaluates the link quality through the computational process of the EGD to eliminate the weak links and then uses the EstD to steer the route request packets toward the general direction of the destination.

A. K. Daniel et al. [7] have proposed a new protocol in wireless mobile heterogeneous networks based on the use of path information, traffic, stability estimation factor, and bandwidth resource information at each node for allocating the route path and buffer. This can handle the hand-off problem of the mobile network. It uses two buffers for the new call and hand-off calls. If there is no channel available instead of dropping them, it will store in the buffer. Whenever the channel is free, it will allocate the packets for communication. This protocol greatly improves the performance of the network.

C. Priyadharshini et al., [21] have proposed a new algorithm, which utilizes the network parameters related to dynamic nature of nodes through energy drain rate and relative mobility estimation rate to predict the node lifetime and link lifetime. The least dynamic route has been selected for forwarding the data packets. Finally, this route lifetime prediction algorithm is implemented in the new protocol environment, which is based on the dynamic source routing (DSR) protocol. This new protocol outperforms the existing protocols like the lifetime prediction routing (LPR) and DSR protocols in terms of throughput, routing failure, routing overhead, packet loss ratio, and packet delivery ratio.

Q. Han et al. [22] have proposed a link availability prediction-based reliable routing for MANETs that takes unpredictable topology changes and frequent link failure into account. The link availability is predicted over a short period of time by estimating the distance between two adjacent nodes. They have derived an analytical expression for link availability based on the relative mobility of the nodes.

Yen Yun-Sheng et al. [23] have proposed a multiconstrained QoS multicast routing method using genetic algorithm. It uses the available resources and minimum computation time in a dynamic environment. By selecting the appropriate values for parameters such as crossover, mutation, and population size, the genetic algorithm improves and tries to optimize the routes.

\section{Work description}

In PSO, every particle is considered as a possible solution to the numerical optimization problem in a D-dimensional 


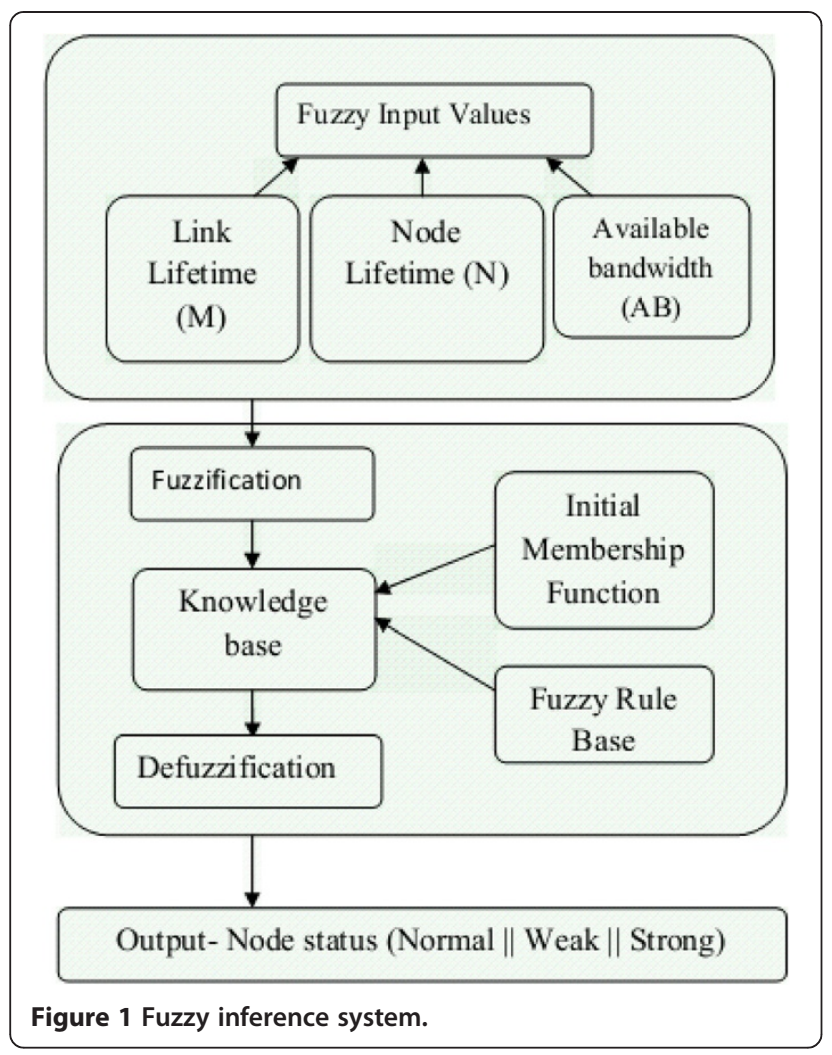

space. In this search space, each particle contains its assigned location and velocity.

Let $P_{\mathrm{i}}$ denote the particle's position, $V_{\mathrm{i}}$ denote the particle's velocity, $L_{\mathrm{bp}}$ be the local memory space, and $G_{\text {bp }}$ be the global memory space.

In addition, each particle contains a local memory space $\left(L_{\mathrm{bp}}\right)$ to store the best position experienced by the particle until then. Each particle contains a global memory space for storing the best global position experienced by the particle until then. Using this information, the velocity of the particle can be estimated using
Equation 1. Equation 2 gives the updated position of the particle.

$$
\begin{aligned}
& V_{\mathrm{i}}=V_{\mathrm{i}}+\gamma_{1} * \operatorname{rand} *\left(L_{\mathrm{bpi}}-P_{\mathrm{i}}\right)+\gamma_{2} * \operatorname{rand} *\left(G_{\mathrm{bp}}-P_{\mathrm{i}}\right) \\
& P_{\mathrm{i}}=P_{\mathrm{i}}+V_{\mathrm{i}}
\end{aligned}
$$

where $\gamma_{1}$ and $\gamma_{2}$ are the constants that estimate the relative influences of the personal and social experiences.

$$
\begin{aligned}
\text { rand }= & \text { Uniformly distributed random number } \\
& \text { in the range of }[0,1]
\end{aligned}
$$

In Equation 1, an inertia factor $(\sigma)$ is introduced, which regulates the velocity and enhances the search accuracy of the particle over time.

$$
\begin{aligned}
V_{\mathrm{i}}= & \sigma * V_{\mathrm{i}}+\gamma_{1} * \operatorname{rand} *\left(L_{\mathrm{bpi}}-P_{\mathrm{i}}\right)+\gamma_{2} \\
& * \operatorname{rand} *\left(G_{\mathrm{bp}}-P_{\mathrm{i}}\right)
\end{aligned}
$$

For efficient control and velocity constraint, a constriction factor $Q$ is introduced in Equation 3.

$$
\begin{gathered}
V_{\mathrm{i}}=Q *\left(\sigma * V_{\mathrm{i}}+\gamma_{1} * \operatorname{rand} *\left(L_{\mathrm{bpi}}-P_{\mathrm{i}}\right)+\gamma_{2}\right. \\
\left.* \operatorname{rand} *\left(G_{\mathrm{bp}}-P_{\mathrm{i}}\right)\right)
\end{gathered}
$$

$$
\text { where } Q=\frac{2}{\left|2-\gamma-\sqrt{\gamma^{2}-4 \gamma}\right|} \gamma=\gamma_{1}+\gamma_{2}
$$

\subsection{Estimation of metrics}

\subsubsection{Link lifetime}

Link lifetime or link availability is defined as the probability that a link will be continuously available for a specified period of time. The link availability can be predicted accurately over a short period of time, by estimating the distance between two nodes [21].

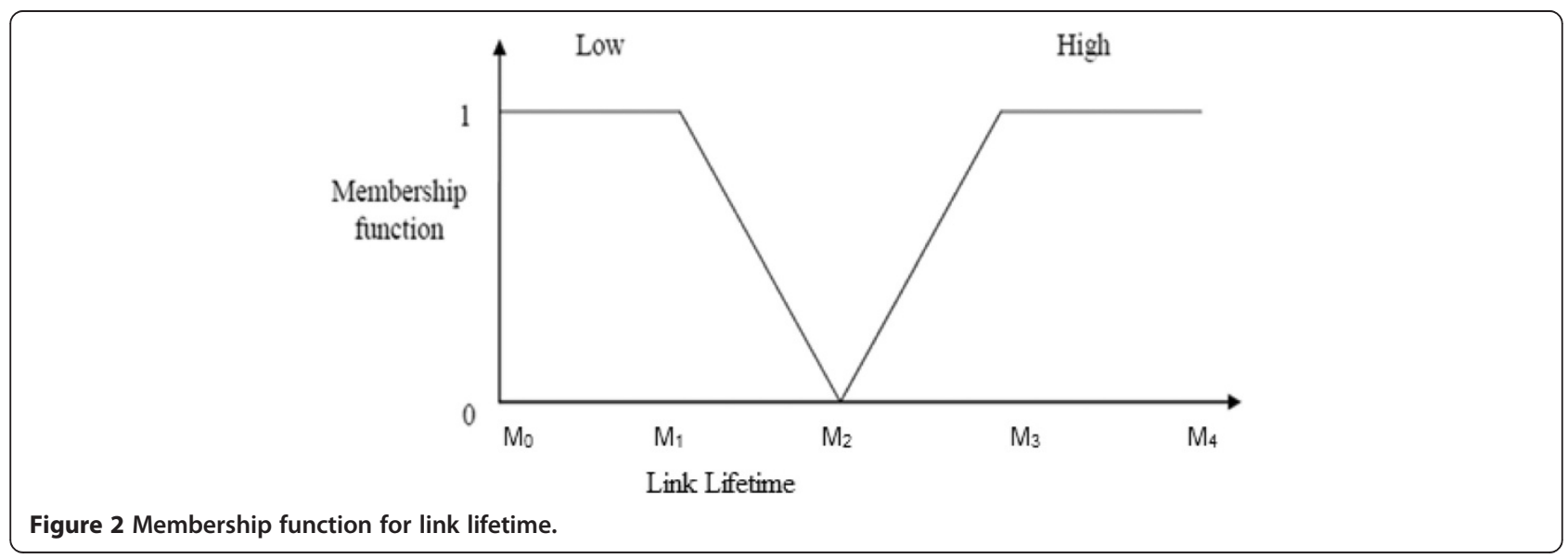




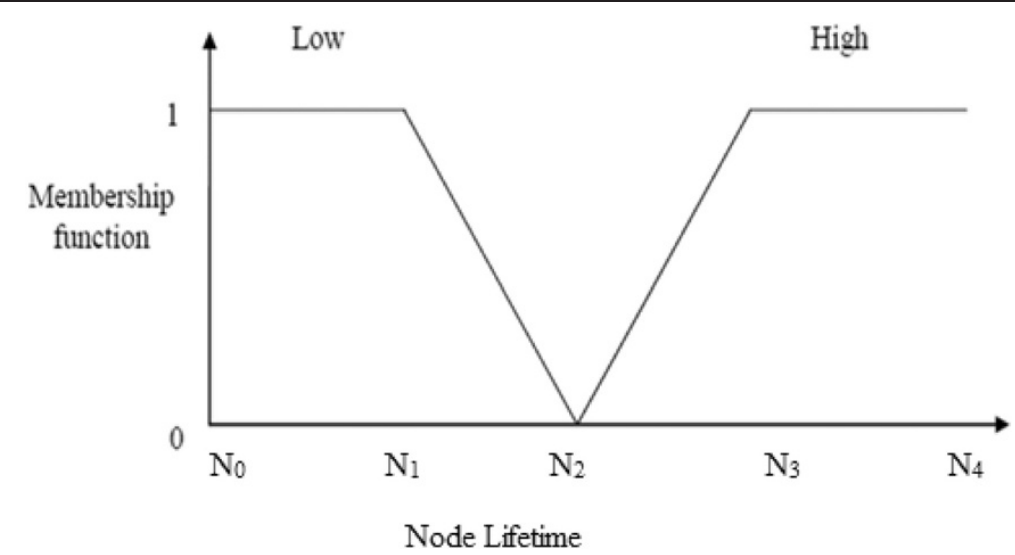

Figure 3 Membership function for node lifetime.

Let $M_{\mathrm{i}}$ represent the link, $x_{\mathrm{i}}$ be the connection, $\mathrm{LT}_{\mathrm{xi}}$ the connection lifetime, $N_{\mathrm{i}-1}$ and $N_{\mathrm{i}}$ be the adjacent nodes, and $B_{N \mathrm{i}}$ and $B_{N \mathrm{i}-1}$ be the battery lifetime of the node $N_{\mathrm{i}}$.

The connection lifetime $\left(\mathrm{LT}_{x \mathrm{i}}\right)$ depends on the relative mobility and distance among the nodes $N_{\mathrm{i}-1}$ and $N_{\mathrm{i}}$ at time $t$. The link lifetime $\left(\mathrm{LT}_{M \mathrm{i}}\right)$ is estimated using the following expression:

$$
\mathrm{LT}_{M \mathrm{i}}=\min \left(\mathrm{LT}_{\mathrm{xi}}, \mathrm{B}_{N \mathrm{i}-1}, \mathrm{~B}_{\mathrm{Ni}}\right)
$$

Thus, the lifetime of route $\mathrm{R}$ is defined as the minimum value of the lifetime of both nodes and connections involved in route $R$.

\subsubsection{Node lifetime}

The nodes may exist in two states such as active and inactive modes. The active mode node drains more energy that results in shorter lifetime than the inactive mode node. Therefore, the node lifetime routing depends upon the energy state of nodes such as residual energy and energy drain rate [21].

Let $R E_{\mathrm{i}}$ be the residual energy of the $N_{\mathrm{i}}, E D_{\mathrm{i}}$ be the energy depletion rate of $N_{\mathrm{i}}$ and $T$ be the duration in seconds.

The node lifetime is estimated using Equation 6:

$$
\mathrm{LT}_{N \mathrm{i}}=\mathrm{RE}_{i}^{n T} / E D_{i}^{n}, \quad t \in[n T,(n+1) T]
$$

\subsubsection{Available bandwidth}

Every node is in charge for estimating the available bandwidth on its link. For a given node [24],

let $\beta$ be the available bandwidth and $C$ be the link capacity associated with one-hop neighbor i.

$A_{\mathrm{R}}$ is the cumulative assigned rates for all incoming and outgoing flows.

Hence, the sum of the assigned incoming and outgoing flow rates and available bandwidth on the link should be equal to the capacity of the link i. This can be expressed as

$$
A_{\mathrm{Rij}}+\beta_{\mathrm{i}}=C_{\mathrm{i}}
$$

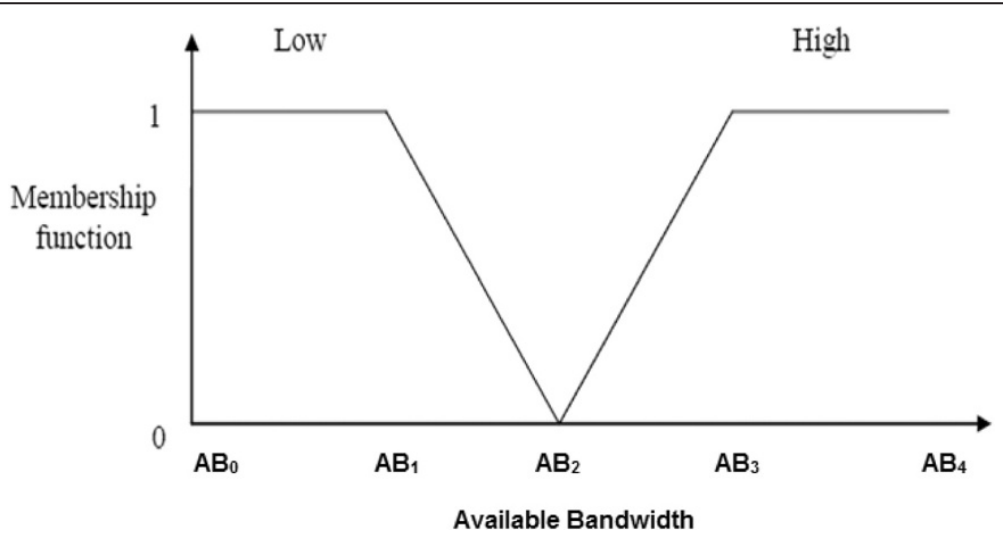

Figure 4 Membership function for available bandwidth. 


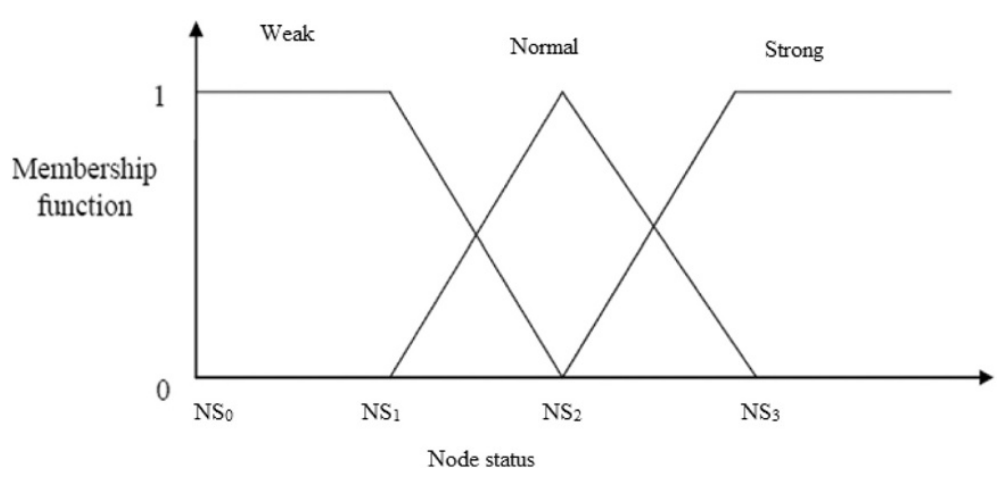

Figure 5 Membership function for node status.

The link capacity is measured and available bandwidth is defined by the following equation:

$$
\beta_{\mathrm{j}} \triangleq \max \left\{0, C_{\mathrm{j}} A_{\mathrm{R}_{\mathrm{ij}}}\right\}
$$

\subsection{PSO-based lifetime prediction algorithm}

Our particle swarm optimization-based proposed algorithm predicts the link lifetime and node lifetime, available bandwidth based on the parameters such as relative mobility of nodes, energy drain rate and link capacity, respectively. It is described below.

\section{Step 1}

When the nodes are deployed in the network, swarm particles $\left(\mathrm{SP}_{\mathrm{i}}\right)$ are initialized such that the particle's position is randomly dispersed in space. Each $\mathrm{SP}_{\mathrm{i}}$ represents a search window equivalent to the node's position and velocity $\left(P_{\mathrm{i}}, V_{\mathrm{i}}\right)$.

\section{Step 2}

Each $\mathrm{SP}_{\mathrm{i}}$ monitors certain parameters of each node such as node lifetime, link lifetime, and available bandwidth.

\section{Step 3}

Based on the monitored parameters, fitness function $\left(F_{\mathrm{i}}\right)$ of each particle is estimated as per Equation 9:

$$
F_{\mathrm{i}}=\left(\alpha_{1} * \mathrm{LT}_{M \mathrm{i}}\right)+\left(\alpha_{2} * \mathrm{LT}_{N \mathrm{i}}\right)+\left(\alpha_{3} * \beta_{\mathrm{i}}\right)
$$

where $\alpha_{1}, \alpha_{2}$, and $\alpha_{3}$ are the weight values.

\section{Table 1 Fuzzy rules for the determining output}

\begin{tabular}{llllc}
\hline S. number & $\boldsymbol{M}$ & $\boldsymbol{N}$ & AB & NS \\
\hline 1 & Low & Low & Low & Weak \\
2 & Low & Low & High & Weak \\
3 & Low & High & Low & Weak \\
4 & Low & High & High & Normal \\
5 & High & Low & Low & Weak \\
6 & High & Low & High & Normal \\
7 & High & High & Low & Normal \\
8 & High & High & High & Strong \\
\hline
\end{tabular}

\section{Step 4}

The local best $\left(L_{\mathrm{bp}}\right)$ and global best $\left(G_{\mathrm{bp}}\right)$ value of fitness and position of each particle is estimated.

\section{Step 5}

Update the position of $L_{\mathrm{bp}}$ and $G_{\mathrm{bp}}$ according to the following condition

i. If $F_{\mathrm{i}}>F_{\mathrm{i}}\left(L_{\mathrm{bpi}}\right)$

Then

Update the position of $L_{\mathrm{bp}}$ with the fitness value $F_{\mathrm{i}}$ End if

ii. If $F_{\mathrm{i}}>F_{\mathrm{i}}\left(G_{\mathrm{bpi}}\right)$

Then

Update the position of $G_{\mathrm{bp}}$ with fitness value $F_{\mathrm{i}}$ End if

\section{Step 6}

Update the velocity and position of each particle using Equations 1 and 2.

\section{Step 7}

The value updated in the global best particle is considered as the best-predicted value.

\section{Step 8}

The predicted link lifetime, node lifetime, and available bandwidth are fuzzified and fuzzy rules are formed to decide the type of node whether it is a weak, normal, or strong node.

\subsection{Fuzzy-based node status estimation}

This technique involves the detection of the node's status by fuzzy logic technique. The steps to determine the fuzzy rule-based interference are as follows:

- Fuzzification: In Fuzzification, the crisp inputs are obtained from the selected input variables and then the degrees to which the inputs belong to each of the suitable fuzzy set are estimated. 

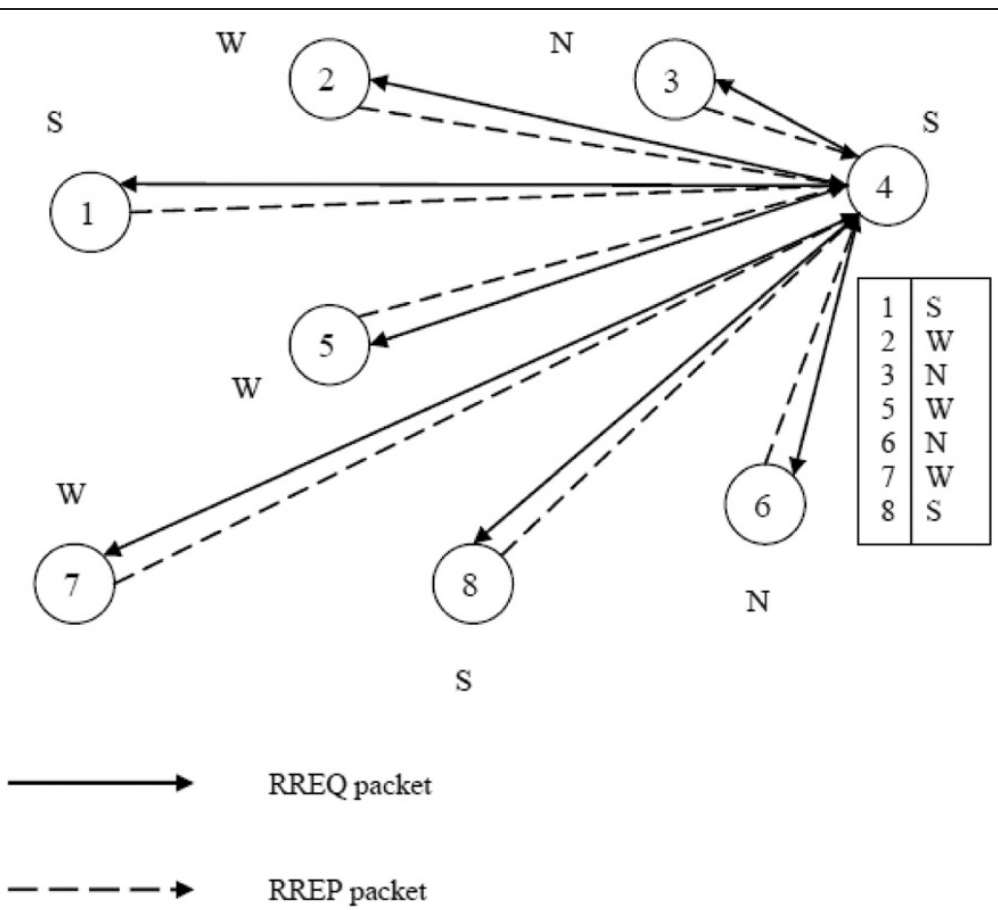

Figure 6 Fuzzy-based selection of node.

- Rule evaluation: The fuzzified inputs are taken and applied to the antecedents of the fuzzy rules. It is then applied to the consequent membership function.

- Aggregation of the rule outputs: This involves merging of the output of all rules.
- Defuzzification: The merged output of the aggregate output fuzzy set is the input for the defuzzification process and a single crisp number is obtained as output.

Initially, the fuzzy logic engine analyzes each node for the detection of the node status such as normal $(\mathrm{N})$,

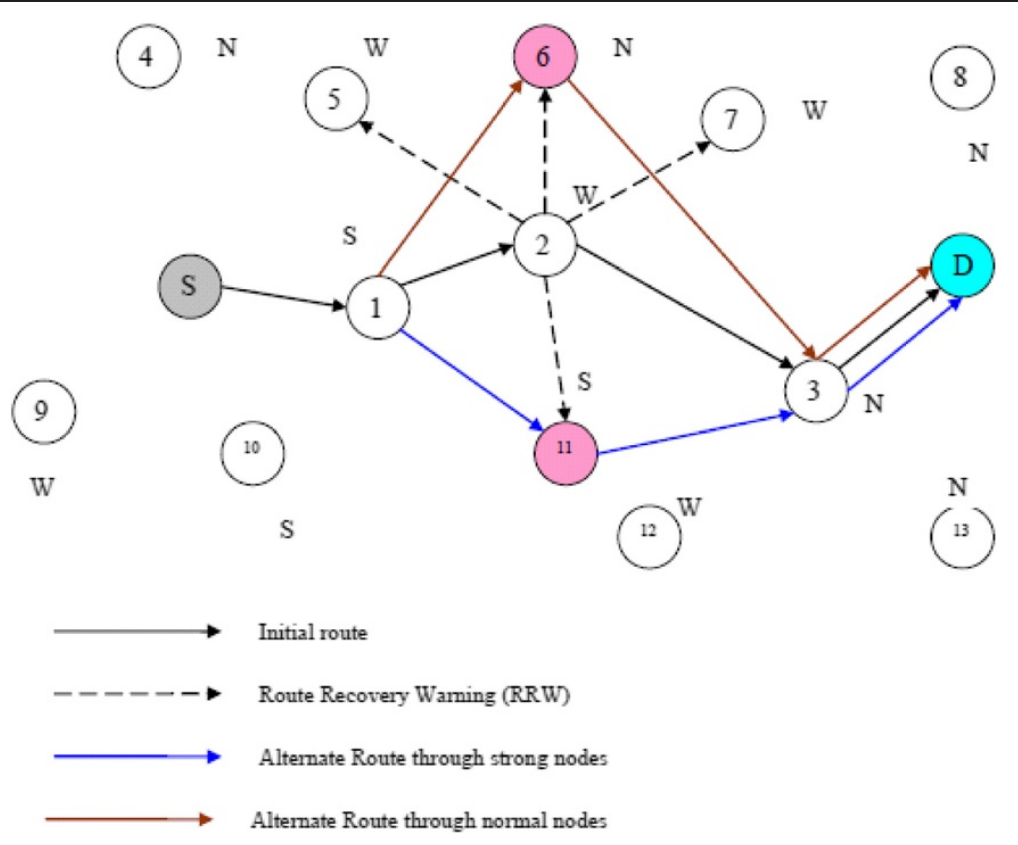

Figure 7 Route recovery process. 
Table 2 Simulation parameters

\begin{tabular}{lc}
\hline & Value \\
\hline Number of nodes & 100 \\
Area size & $1,000 \times 1,000$ \\
Mac & 802.11 \\
Routing protocol & PNLP \\
Simulation time & $50 \mathrm{~s}$ \\
Traffic source & CBR \\
Packet size & $250 \mathrm{~kb}$ \\
Rate & $150 \mathrm{~m}$ \\
Transmission range & 10 to $50 \mathrm{~m} / \mathrm{s}$ \\
Speed of nodes & $0.395 \mathrm{~W}$ \\
Transmit power & $0.660 \mathrm{~W}$ \\
Receiving power & $0.035 \mathrm{~W}$ \\
Idle power & $10.1 \mathrm{~J}$ \\
Initial energy & \\
\hline
\end{tabular}

weak (W) and strong (S) based on predicted parameters such as link lifetime $\left(\mathrm{LT}_{M}\right)$, node lifetime $\left(\mathrm{LT}_{N}\right)$, and available bandwidth $(\beta)$.

The fuzzy inference system is illustrated using Figure 1.

\subsubsection{Fuzzification}

This involves fuzzification of input variables such as link lifetime $(M)$, node lifetime $(N)$, and available bandwidth $(\mathrm{AB})$ and these inputs are given a degree to appropriate fuzzy sets. The output crisp inputs are the combination of $M, N$, and $B$. We take two possibilities, high and low, for $M, N$, and $\mathrm{AB}$.

Figures 2, 3, 4, and 5 show the membership function for the input and output variables. Due to the computational efficiency and uncomplicated formulas, the triangulation functions are utilized. These triangular functions are widely used in real-time applications. Also, a positive impact is offered by this design of membership function.

In Table $1, M, N$, and $\mathrm{AB}$ are given as inputs and the output NS represents the status of each node. The eight fuzzy sets are defined with the combinations presented in Table 1.

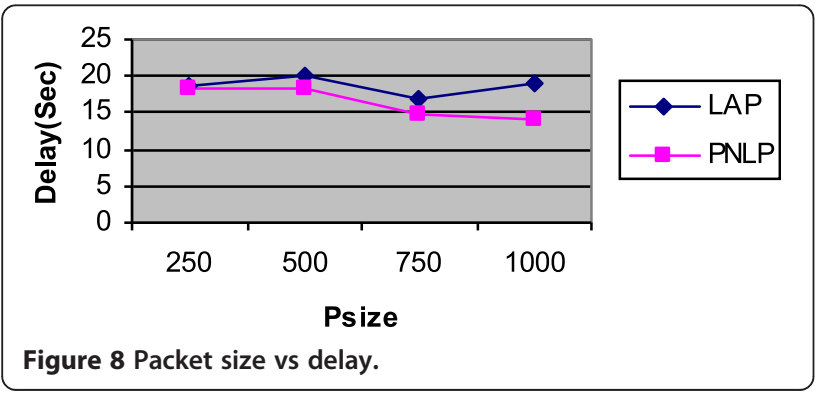

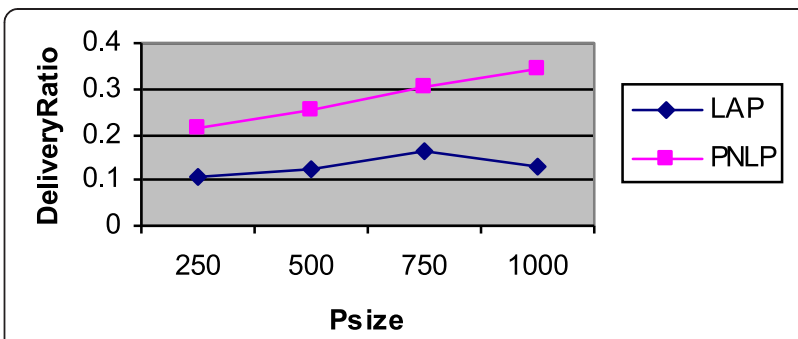

Figure 9 Packet size vs delivery ratio.

Table 1 demonstrates the rules of the fuzzy inference system. This illustrates the function of the inference engine and method by which the outputs of each rule are combined to generate the fuzzy decision.

If $M, N$, and $\mathrm{AB}$ are low, the node status will be weak.

If $M$ and $N$ are low and $\mathrm{AB}$ is high, the node status will be weak.

If $M$ and $\mathrm{AB}$ are low and $N$ is high, the node status will be weak.

If $M$ is low and $N$ and $\mathrm{AB}$ are high, the node status will be normal.

If $M$ is high and $N$ and $\mathrm{AB}$ are low, the node status will be weak.

If $M$ and $\mathrm{AB}$ are high and $N$ is low, the node status will be normal.

If $M$ and $N$ are high and $\mathrm{AB}$ is low, the node status will be normal.

If $M, N$, and $\mathrm{AB}$ are high, the node status will be strong.

\subsubsection{Defuzzification}

The technique by which the crisp values are extracted from a fuzzy set as a representation value is referred to as defuzzification. The centroid of the area scheme is taken into consideration for defuzzification during fuzzy decision-making process. The formula (10) describes the defuzzifier method.

$$
\text { Fuzzy_cost }=\left[\sum_{\text {allrules }} Z_{i} * \lambda\left(z_{i}\right)\right] /\left[\sum_{\text {allrules }} \lambda\left(z_{i}\right)\right]
$$

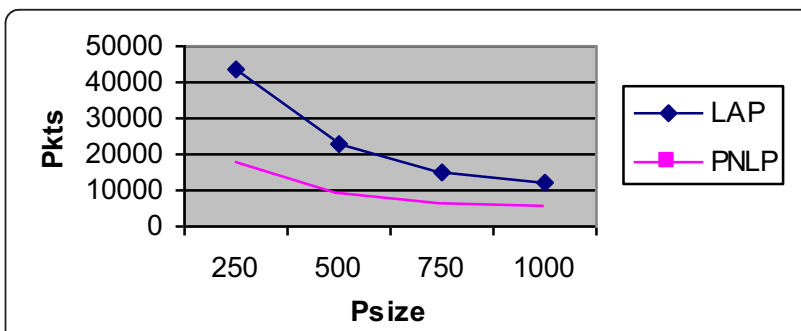

Figure 10 Packet size vs drop. 


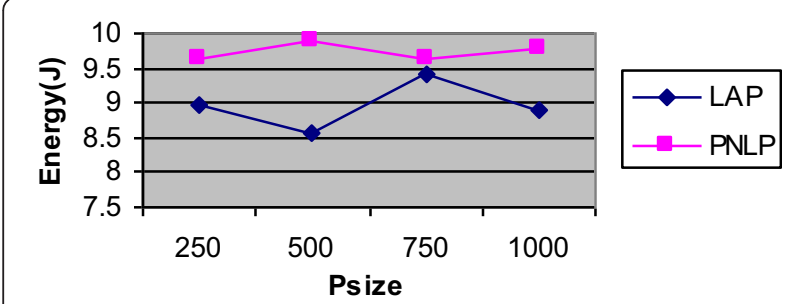

Figure 11 Packet size vs residual energy.

Fuzzy_cost is used to specify the degree of decision making, $z_{\mathrm{i}}$ is the fuzzy all rules, and variable and $\gamma\left(z_{\mathrm{i}}\right)$ is its membership function. The output of the fuzzy cost function is modified to crisp value as per this defuzzification method.

\subsection{Route discovery}

As an outcome of fuzzy decision rules, the node status can be considered as weak, normal, and strong. Initially, we assign each node with the status normal. This estimated decision is exchanged among all neighbors using HELLO messages. When the source node sends data to the destination, data packets are transmitted through nodes that are in the routing table.

In Figure 6, nodes 1, 2, 3, 4, 5, 6, 7, and 8 are assigned with particular node status. Nodes 1,8 , and 4 are strong nodes, nodes 2,5 , and 7 are weak nodes, and nodes 3 and 6 are normal nodes. Here, we assume that the node 4 sends RREQ request to the other nodes to maintain a route table. All the nodes reply their node status in the RREP packet to the node 4 .

\subsection{Route maintenance}

Before a node transmits the data to the next node, it checks the status of that node. If the status is normal or strong, it will transmit the packet to the next node. If the status of the successor node is weak, it will send a route recovery warning (RRW) message to all neighboring nodes. Upon receiving the RRW packet, the neighboring nodes look around to find strong nodes. If it finds strong nodes, they will initiate the local route recovery process by changing the route to the strong nodes. If they cannot find

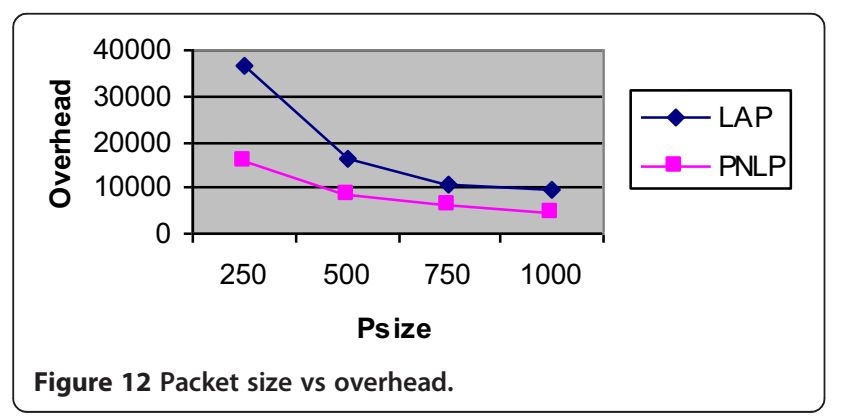

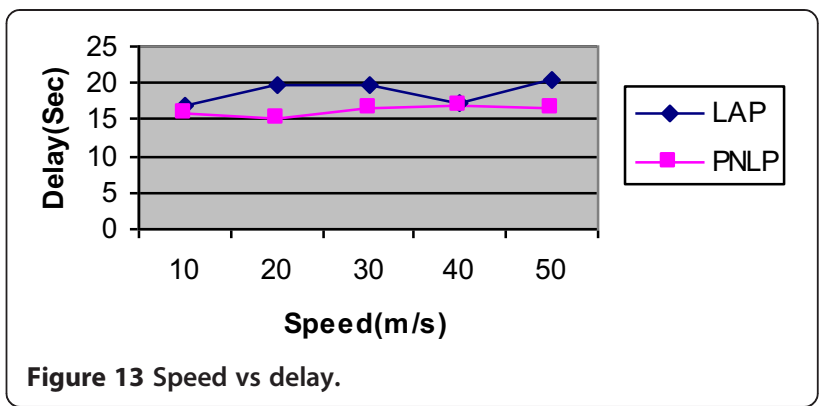

any strong nodes, they will initiate the route recovery process through normal nodes.

In Figure 7, the route recovery process is explained. Here, a primary path is set from the source node to the destination via $\mathbf{S} \rightarrow \mathbf{1} \rightarrow \mathbf{2} \rightarrow \mathbf{3} \rightarrow \mathbf{D}$. Each node checks its routing table before it transmits the data. Here, node 1 checks the status of its next hop node, which is found to be weak. Therefore, node 1 sends a RRW to all its neighboring nodes $5,6,7$, and 11 . Node 2 finds that node 11 status is strong and thus alternate path is provided through node 11, i.e., $\mathbf{S} \rightarrow \mathbf{1} \rightarrow \mathbf{1 1} \rightarrow \mathbf{3} \rightarrow \mathbf{D}$. When a strong node is found in the neighborhood, node 2 will select the normal node for providing the alternate path. Here, path $\mathbf{S} \rightarrow \mathbf{1} \rightarrow \mathbf{6} \rightarrow \mathbf{3} \rightarrow \mathbf{D}$ is established.

\subsubsection{Advantages of the proposed approach}

1) Since the lifetime factor of the node and link is taken into account for routing, data loss is reduced.

2) The route failure is greatly minimized thereby reducing the overhead.

3) PSO algorithm is easier to implement and very efficient in the global search.

\section{Simulation results}

The performance of our PSO-based node and link lifetime prediction algorithm (PNLP) is evaluated through NS2 [25] simulation. A random network deployed in an area of $1,000 \times 1,000 \mathrm{~m}$ is considered. Initially, 100 sensor nodes are placed in square grid area by placing each sensor in a $50 \times 50$ grid cell. The sink is assumed to be situated $100 \mathrm{~m}$ away from the above-specified area. In the simulation, the

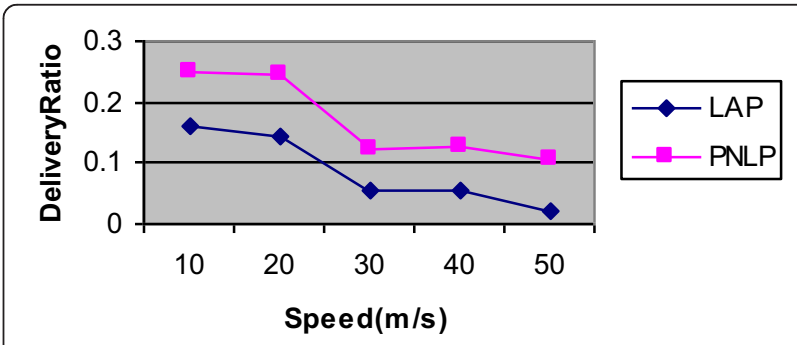

Figure 14 Speed vs delivery ratio. 
channel capacity of mobile hosts is set to the same value: 2 Mbps. The simulated traffic is CBR with UDP source and sink. The number of sources is fixed as 4 around a phenomenon. Table 2 summarizes the simulation parameters used.

\subsection{Performance metrics}

The performance of PNLP technique is compared with the link availability prediction (LAP)-based routing protocol [22].

The performance is evaluated mainly, according to the following metrics:

- Average packet delivery ratio: It is the ratio of the number of packets received successfully and the total number of packets transmitted.

- Drop: It is the number of packets dropped during the transmission.

- Delay: It refers to the average end-to-end delay of packets.

- Energy: It is the average energy consumed for the data transmission.

- Overhead: It is the ratio between the number of packets rejected and number of packets sent.

\subsection{Results}

\subsubsection{Based on packet size}

The packet size is varied as $250,500,750$, and 1,000 bytes and the performance metrics are measured for the two protocols.

Figure 8 shows the delay which occurred for both the techniques when the packet size is increased from 250 to 1,000 bytes. Since PNLP predicts the link lifetime more accurately than LAP, the delay is $0.2 \%$ less for it.

The packet drop and delivery ratio for both PNLP and LAP are shown in Figures 9 and 10, respectively. As the packet size is increasing, the data-sending rate will be reduced that results in reduced packet loss. Hence, the packet drop decreases and delivery ratio gradually increases, when the packet size is increased. However, PNLP has $1.3 \%$ reduced packet drops, when compared to LAP, since it proactively avoids the failure of nodes

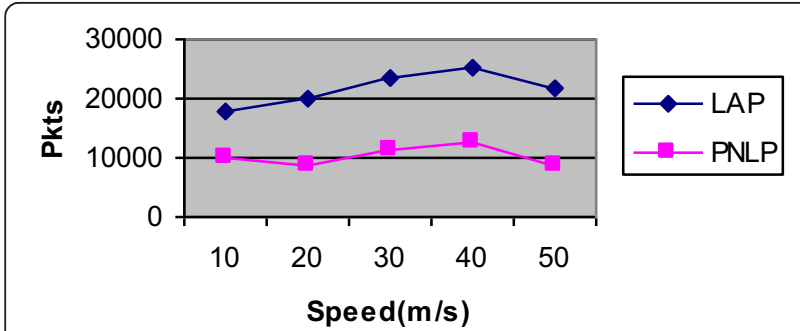

Figure 15 Speed vs drop.

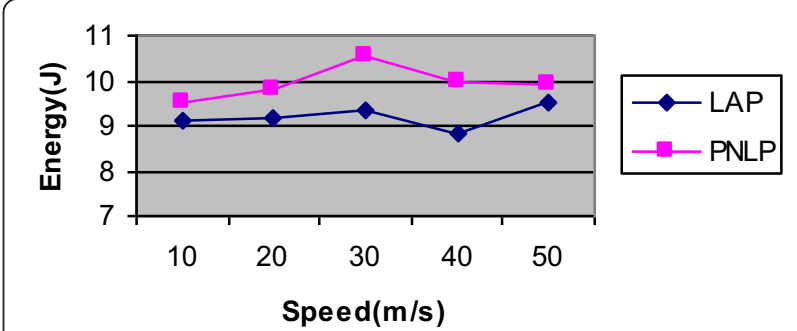

Figure 16 Speed vs residual energy.

also. Hence, the packet delivery ratio of PNLP is $0.5 \%$ higher than LAP.

Figure 11 shows the residual energy for both the techniques when the packet size is increased from 250 to 1,000 bytes. It can be seen that PNLP has $0.08 \%$ higher energy when compared to LAP since it considers residual energy as one parameter for node status.

The overhead occurred during the routing process is depicted in Figure 12. When the packet size is increased, it results in reduced congestion and hence alternate routing will not be triggered often. Therefore, the overhead is decreasing linearly as shown in the figure. Nevertheless, PNLP has 1.01\% lower overhead when compared to LAP.

\subsubsection{Based on speed}

Next, the node's speed is varied as $10,20,30,40$, and $50 \mathrm{~m} / \mathrm{s}$ and performance metrics are measured for the two protocols.

Figure 13 shows that the delay occurred for both the techniques when the speed is increased from 10 to $50 \mathrm{~m} / \mathrm{s}$. As we can see from the figure, the delay begins to increase when the speed is increased since the chances of route breakage are more at high speed. Since PNLP predicts link lifetime more accurately than LAP, the delay is $0.2 \%$ less for it.

The packet drop and packet delivery ratio for both PNLP and LAP are depicted in Figures 14 and 15, respectively. As the speed increases, it maximizes the chances of route failures. Hence, the packet drop begins to increase and delivery ratio gradually decreases. However, PNLP has $1.25 \%$ reduced packet drops,

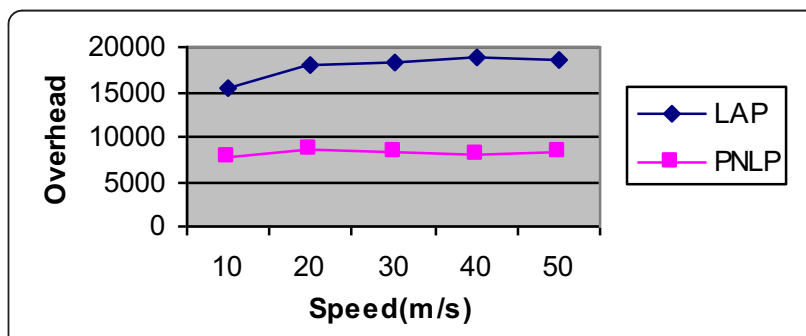

Figure 17 Speed vs overhead. 
when compared to LAP, since it accurately predicts and avoids the failure of nodes proactively. Hence, the packet delivery ratio of PNLP is $0.6 \%$ higher than LAP.

Figure 16 shows the residual energy both the techniques when the speed is increased from 10 to $50 \mathrm{~m} / \mathrm{s}$. It can be seen that PNLP has $0.08 \%$ higher residual energy when compared to LAP, since it considers residual energy as one parameter for node status.

The overhead occurred during the routing process is depicted in Figure 17. When speed is increased, it results in increased route failures and hence the possibility of alternate routing will be more. So the overhead is slightly increasing for LAP and almost constant for PNLP, as shown in the figure. However, PNLP has 1.02\% lower overhead when compared to LAP since the congestion and alternate route information are not exchanged prior in PNLP.

\section{Conclusion}

In this manuscript, a particle swarm optimization (PSO)-based lifetime prediction algorithm for route recovery in MANET has been proposed. This technique predicts the lifetime of link and node in the available bandwidth based on the parameters like relative mobility of nodes and energy drain rate, etc.. Using predictions, the parameters are fuzzified and fuzzy rules have been formed to decide on the node status. This information is made to exchange among all the nodes. Thus, the status of the every node is verified before data transmission. Even for a weak node, the performance of a route recovery mechanism is made in such a way that corresponding routes are diverted to the strong nodes. With the aid of the simulated results, the minimization of data loss and communication overhead is using PSO prediction.

\section{Competing interests}

The authors declare that they have no competing interests.

\section{Authors' information}

Dr. V. Rhymend Uthariaraj Professor \& Director, Ramanujan Computing Centre v.rhymenduthariaraj@yahoo.com.

\section{Author details}

${ }^{1}$ Research Scholar, Department of Information \& Communication Engineering, Anna University, Chennai-25, India. ${ }^{2}$ Ramanujan Computing Centre, Anna University, Chennai-25, India.

Received: 27 July 2013 Accepted: 20 May 2014

Published: 3 July 2014

\section{References}

1. J Yogendra Kumar, V Rakesh Kumar, Energy level accuracy and life time increased in mobile ad-hoc networks using OLSR. Int. J. adv. Res. Comput. Sci. Softw. Eng 2, 7 (2012)

2. K Ajay, MS Sheethal, J Shany, P Priya, Optimum route life time prediction of trusted dynamic mobile nodes in large scale MANETs. Inter. J. Ad hoc, Sensor \& Ubiquitous Computing 3, 3 (2012)

3. E Natsheh, S Khatun, AB Jantan, S Subramaniam, Fuzzy metric approach for route lifetime determination in wireless ad hoc networks. Int. J of Ad Hoc and Ubiquitous Computing 3, 1 (2007)
4. I Ahmed, KE Tepe, BK Singh, Reliable coverage area based link expiration time (LET) routing metric for mobile ad hoc networks, in Ad Hoc Networks (Springer, Berlin Heidelberg, 2010), pp. 466-476

5. XM Zhang, BW En, JX Jing, KS Dan, An estimated distance-based routing protocol for mobile ad hoc networks. IEEE Trans on Vehicular Tech 60(7), 3473-3484 (2011)

6. X Wang, AV Vasilakos, M Chen, Y Liu, TT Kwon, A survey of green mobile networks: opportunities and challenges. MONET 17(1), 4-20 (2012)

7. AK Daniel, R Singh, JP Saini, Swarm intelligence based routing technique for call blocking in heterogeneous mobile adhoc network using link stability factor and buffering technique for QoS. Int. J. Res. Rev. Comput. Sci 2(1), 65-72 (2011)

8. G Wei, Y Ling, B Guo, B Xiao, AV Vasilakos, Prediction-based data aggregation in wireless sensor networks: combining grey model and Kalman filter. Comput. Commun 34, 793-802 (2011)

9. A Vasilakos, C Ricudis, K Anagnostakis, W Pedryca, W Pedryca, Evolutionary-fuzzy prediction for strategic QoS routing in broadband networks, in The 1998 IEEE International Conference on Fuzzy Systems Proceedings, Anchorage, AK, 4-9 May 1998, vol. 2, 1998, pp. 1488-1493

10. P Rajeswari, Estimation of node lifetime based on dynamic nature routing in MANET. Inter. J Adv. Info. Sci. Tech. 9, 9 (2013)

11. BY Narendra, CH Suresh Babu, Route recovery schemes for efficient MANET. Inter. J. Emerg. Trends. Eng. Dev 1, 3 (2013)

12. U Shuchita, G Charu, Node disjoint multipath routing considering link and node stability protocol: a characteristic evaluation. Inter. J Com Sci. Issues 7(1), 2 (2010)

13. Z Yuanyuan, X Kai, L Deshi, W Athanasios, Directional routing and scheduling for green vehicular delay tolerant networks. Wireless Netw. 19(2), 161-173 (2013)

14. S Thrasyvoulos, NBR Rao, T Thierry, O Katia, V Athanasios, Routing for disruption tolerant networks: taxonomy and design. Wireless Netw. 16(8), 2349-2370 (2010)

15. N Chilamkurti, S Zeadally, A Vasilakos, V Sharma, Cross-layer support for energy efficient routing in wireless sensor networks. J. Sensors 2009, (2009)

16. Y Ahmet Sekercioglu, P Andreas, V Athanasios, Computational intelligence in management of ATM networks: a survey of current state of research. Soft. Comput. 5(4), 257-263 (2001)

17. P Andreas, G Stylianou, CS Pattichis, A Sekercioglu, A Vasilakos, Bandwidth allocation for virtual paths (BAVP): investigation of performance of classical constrained and genetic algorithm based optimization techniques, in INFOCOM 2000, Tel Aviv, ed. by, 26-30 Mar 2000, pp. 1501-1510

18. S Waseem, AK Farrukh, BS Abdul, Weighted clustering using comprehensive learning particle swarm optimization for mobile ad hoc networks. Inter. J. Future Generation Commun. Netw 3, 1 (2010)

19. A Shahriar, A Sima Uyar, A novel particle swarm optimization algorithm, in The 10th International Conference on Artificial Evolution, ed. by (Angers, France, 24 October 2011)

20. C Priyadharshini, K Thamarai Rubini, PSO based route lifetime prediction algorithm for maximizing network lifetime in MANET, in 2012 International Conference on Recent Trends in Information Technology (ICRTIT), Chennai, Tamil Nadu, ed. by, 19-21 Apr 2012, pp. 270-275

21. C Priyadharshini, K ThamaraiRubini, Predicting route lifetime for maximizing network lifetime in MANET, in 2012 International Conference on Computing, Electronics and Electrical Technologies (ICCEET), Nagercoil, Tamil Nadu, ed. by, 21-22 Mar 2012, pp. 792-797

22. Q Han, Y Bai, L Gong, W Wu, Link availability prediction-based reliable routing for mobile ad hoc networks, in The 10th IET Communications 2011, ed. by, vol. 5, 2011, pp. 2291-2300. doi:10.1049/iet-com.2010.0946

23. Y Yun-Sheng, C Han-Chieh, C Ruay-Shiung, V Athanasios, Flooding-limited and multi-constrained QoS multicast routing based on the genetic algorithm for MANETs. Math. Comput. Model. 53(11-12), 2238-2250 (2011)

24. KS Vishnu, SB Sarita, Agent based bandwidth reservation routing technique in mobile ad hoc networks. Inter. J. Adv. Comput Sci. Appl 2, 12 (2011)

25. ISI, Network simulator-ns-2. http://www.isi.edu/nsnam/ns

\section{doi:10.1186/1687-1499-2014-107}

Cite this article as: Manickavelu and Vaidyanathan: Particle swarm optimization (PSO)-based node and link lifetime prediction algorithm for route recovery in MANET. EURASIP Journal on Wireless Communications and Networking 2014 2014:107. 